\title{
Construction of shape features for the representation of full-field displacement/strain data
}

\author{
Weizhuo Wang ${ }^{1, a}$, John E Mottershead ${ }^{1, b}$, Amol Patki $^{2, c}$ and \\ Eann A Patterson ${ }^{2, d}$ \\ ${ }^{1}$ Department of Engineering, University of Liverpool, Liverpool, UK, L69 3GH \\ ${ }^{2}$ Department of Mechanical Engineering, Michigan State University, East Lansing, MI, USA \\ ${ }^{a}$ wangweizhuo@gmail.com ${ }^{\text {bj.e.mottershead@liverpool.ac.uk }}$ \\ c patkiamo@egr.msu.edu ${ }^{\mathrm{d}}$ eann@egr.msu.edu
}

Keywords: full-field strain pattern, shape features, Zernike moment, model updating

\begin{abstract}
The achievement of high levels of confidence in finite element models involves their validation using measured responses such as static strains or vibration mode shapes. A huge amount of data with a high level of information redundancy is usually obtained in both the detailed finite element prediction and the full-field measurements so that achieving a meaningful validation becomes a challenging problem. In order to extract useful shape features from such data, image processing and pattern recognition techniques may be used. One of the most commonly adopted shape feature extraction procedures is the Fourier transform in which the original data may be expressed as a set of coefficients (coordinates) of the decomposition kernels (bases) in the feature space. Localised effects can be detected by the wavelet transform. The acquired shape features are succinct and therefore simplify the model validation, based on the full-field data, allowing it to be achieved in a more effective and efficient way. In this paper, full-field finite element strain patterns of a plate with a centred circular hole are considered. A special set of orthonormal shape decomposition kernels based on the circular Zernike polynomials are constructed by the Gram-Schmidt orthonormalization process. It is found that the strain patterns can suitably be represented by only a very small number of shape features from the derived kernels.
\end{abstract}

\section{Introduction}

Numerical model updating [1] with the availability of full-field measurements [2] involves handling a huge amount of data with a high level of redundancy. It is necessary to extract useful information from the full-field responses, e.g. stress/strain, mode shapes etc, in a succinct form. Shape feature extraction $[3,4]$ is one of the feasible ways to tackle such problems.

Simple shape features such as perimeter of boundaries, area, diameter, circularity, orientation and eccentricity describe the shape in a general way. More substantial shape features may be obtained by functional transformations [4]. In this case, the original shape may be expressed as a set of coefficients (coordinates) of the decomposition kernels (bases) in the feature space. The choice of transformation bases is problem dependent. For instance, periodic features can easily be represented by the Fourier transform; Local significance and global approximation can be detected by the wavelet transform and the Zernike moment is especially powerful in the discrimination of circular shapes.

Proper selection of the kernel functions usually results in a small number of significant and effective shape features by the nature of orthonormal kernels. However, the orthonormality holds only if the domain of the shape matches the definition domain of the orthonormal kernels. For example, the circular Zernike decomposition kernels, known as the Zernike polynomials, are orthonormal over the unit circle [6]. When applying the Zernike moment to non-circular structures, modification of the kernels has to be carried out to satisfy the orthonormality. Mahajan [5] 
employed the Gram-Schmidt orthonormalisation (GSO) process to produce a set of orthonormal Zernike annular polynomials. The GSO process is theoretically applicable to any structures.

In this paper, full field strain patterns of a square specimen with a central circular hole are considered. Sets of orthonormal kernels based on the modified Zernike polynomials defined over the specimen are generated by the GSO process. Results show that the full-field strain pattern with thousands pixels can efficiently be described by only 10 shape features by the constructed Zernike kernels.

\section{Construction of shape features - modified Zernike moments}

The general form of transform-based shape features may be expressed as,

$$
\mathfrak{D}_{i}=\int_{\Omega} \mathfrak{K}_{i}^{*}(x, y) \mathcal{S}(x, y) \mathrm{d} x \mathrm{~d} y
$$

where $\mathcal{S}(x, y)$ denotes the continuous shape pattern, $\Omega$ denotes the domain of definition, $\mathfrak{K}_{i}(x, y)_{i=1,2, \ldots}$ are the set of transformation kernels and $*$ denotes the complex conjugate. For instance, Fourier features can be obtained by assigning complex sinusoids as the kernel functions.

When the orthogonal Zernike polynomials $\left\{V_{i, i=1,2, \ldots}\right\}$ defined over a unit circle are adopted as the kernel functions, equation (1) is expressed as

$$
\mathfrak{D}_{Z_{i}}=\int_{x^{2}+y^{2} \leq 1} V_{i}^{*}(x, y) \mathcal{S}(x, y) \mathrm{d} x \mathrm{~d} y
$$

where the Zernike polynomials

$$
V_{i} \equiv V_{n, m}(x, y) \equiv V_{n, m}(\rho, \vartheta)=R_{n, m}(\rho) \mathrm{e}^{\mathrm{i} m \vartheta}
$$

where $R_{n, m}(\rho)$ represents the radial polynomials [7]. Thus, $\mathfrak{D}_{Z_{i}}$ may now be called the Zernike moment descriptor (ZMD).

\section{Gram-Schmidt orthonormalization of the Zernike polynomial over a non-circular domain}

A set of orthonormal Zernike polynomials defined over an arbitrary domain, e.g. a rectangular plate with a circular hole as shown in figure 1, may be determined by Gram-Schmidt orthonormalization (GSO) as

$$
P_{1}^{\prime}=V_{1} \quad \text { and } \quad P_{\ell}^{\prime}=V_{\ell}-\sum_{k=1}^{\ell-1} \operatorname{proj}_{P_{k}^{\prime}}\left(V_{\ell}\right), \text { for } \ell, k \in \mathbb{N} \text { and } \ell \geq 2
$$

where $\mathbb{N}$ is the set of natural numbers, $\operatorname{proj}_{P_{k}^{\prime}}\left(V_{\ell}\right)$ denotes the projection of $V_{\ell}$ onto $P_{k}^{\prime}$ with respect to the definition domain $H(x, y)$ which is a binary (zero or one valued) function that defines the shape of the structure, $V_{\ell}$ is the circular Zernike polynomial; $\left\{P_{1}^{\prime}, P_{2}^{\prime}, \ldots, P_{N}^{\prime}\right\}$ are the Gram-Schmidt orthogonalized arbitrary Zernike polynomials which can further be normalised by $P_{i}=P_{i}^{\prime} /\left\|P_{i}^{\prime}\right\|$. Thus, $\left\{P_{1}, P_{2}, \ldots, P_{N}\right\}$ are the orthonormal arbitrary Zernike polynomials. For example, the function $H_{R C}(x, y)$ for a rectangular plate with a circular hole as shown in Fig 1 can be defined as

$$
H_{R C}(x, y)= \begin{cases}1, & |x| \leq \frac{a}{2} ;|y| \leq \frac{b}{2} \text { and } \sqrt{x^{2}+y^{2}} \geq \varepsilon \\ 0, & \text { otherwise }\end{cases}
$$


Thus, shape features, i.e. the Zernike moment defined over the non-circular structure, can be obtained by substituting the Gram-Schmidt orthonormalized kernels $P_{k}(x, y)$ into equation (1) as

$$
z_{k}=\iint_{H_{R C}} \mathcal{S}(x, y) P_{k}^{*}(x, y) \mathrm{d} x \mathrm{~d} y
$$

where $z_{k}$ is called the $k^{\text {th }}$ Zernike moment of the strain patterns $\mathcal{S}(x, y)$.

By definition, the number of decomposition kernels $\left\{P_{k}, k=1,2, \ldots\right\}$ is infinity and so is the number of shape feature descriptors. It is essential to reduce the number of the descriptors without significant loss of information. Appropriate selection of the kernels according to the problem in hand may result in only a small number of significant descriptors. However, certain criteria must be met to ensure the retained small number of significant shape features is sufficient to represent the original pattern. Comparing the norm of the reconstructed pattern by retaining the significant terms with the original is a feasible criterion [7].

\section{Full-field strain pattern decomposition using the modified Zernike polynomials}

A thin square plate with a circular hole in the centre is modelled. The dimension of the plate is $0.1 \mathrm{~m}$ $\times 0.1 \mathrm{~m}$ and the radius of the hole is $0.01 \mathrm{~m}$. The specimen is modelled by plane stress elements as shown in Fig 2. Elastic-plastic material with isotropic hardening is considered. Tensile pressure ramp loading is applied steadily from time 0.0 to 3.0 seconds to the two side edges. Four particular points in the ramp loading history are shown in Table 1. Thus, the responses at 1.0, 2.0 and 3.0 seconds are considered in this example.

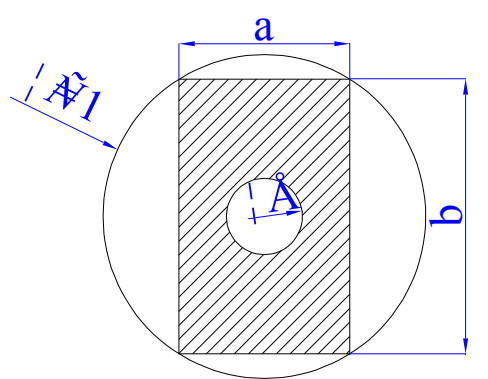

Fig 1. Rectangular test specimen with a circular hole normalised inside an unit circle - constrains:

$$
a^{2}+b^{2}=4 ; \varepsilon<\min \left(\frac{a}{2}, \frac{b}{2}\right)
$$



Fig 2 : FE mesh

Table 1: Ramp Loading (Pressure)

\begin{tabular}{|c|c|c|c|c|}
\hline & & Step 1 & Step 2 & Step 3 \\
\hline Time (Sec) & 0.0 & 1.0 & 2.0 & 3.0 \\
\hline Pressure (MPa) & 0.0 & 80.0 & 160.0 & 240.0 \\
\hline
\end{tabular}

The shape patterns of the maximum principal strain for the three loading stages are shown in the first row of Fig 3. Full-field shape pattern descriptors are not necessarily concentrated on the regions of high strain (such as around the circumference of the central hole) and special measures are therefore necessary to ensure that the shape can be represented using an acceptably small number of descriptors. The continuous strain pattern of the whole structure can be interpolated from the nodal strains using element shape functions. Then the important shape features of these fullfield data may be extracted by the chosen decomposition kernels. An intuitive selection is the Zernike polynomial because the maximum and minimum strains appear in the regions arranged symmetrically around the circumference of the central hole. For example, the $14^{\text {th }}$ circular Zernike polynomials, as shown in Fig 4 (a), exhibit patterns that are arranged similarly to the strain distributions. A set of orthonormal kernels defined over the domain of the specimen can be obtained 
by applying the Gram-Schmidt orthonormalization (GSO) based on the circular Zernike polynomials. The $14^{\text {th }}$ kernel of the GSO-processed Zernike polynomials, denoted as the GSO_Z1 kernel, is shown in Fig 4 (b). It is obvious that the high-strain locations in the GSO_Z1 kernel is not close enough to the centre, when compared to the strain patterns. This problem may be overcome by transforming the radial coordinates of the circular Zernike polynomial before carrying out the GSO. For instance, the radial coordinate $\rho$ in the radial function $R_{n, m}(\rho)$ of equation (3) may be replaced with $\rho^{\prime} \equiv \rho^{\prime}(\rho, v)=\rho^{v}$ where $s \in \mathbb{R}$ and $0<v<1$. Thus, the transformed radial function is expressed as

$$
R_{n, m}^{\prime}(\rho) \equiv R_{n, m}\left(\rho^{\prime}\right)=R_{n, m}\left(\rho^{v}\right)
$$

Taking $\rho^{\prime}=\rho^{0.25}$ for example, another set of orthonormal kernels, denoted as GSO_Z2, can be obtained by applying the GSO process to the coordinate-transformed Zernike polynomials. The $14^{\text {th }}$ GSO_Z2 as illustrated in Fig 4 (c) is closer to the strain patterns than GSO_Z1 for the region near the central hole. However, large values remain in the regions around the outside edges of the GSO_Z2 kernels, which are unrepresentative of the strain distributions. A decaying weight function $w(\rho, \bar{t})$ may be applied to the radial functions of the coordinate-transformed Zernike polynomials to reduce this effect,

$$
R_{n, m}^{w}(\rho, t) \equiv w(\rho, t) R_{n, m}^{\prime}(\rho)=\rho^{t} R_{n, m}^{\prime}(\rho), \quad t \in \mathbb{R}^{-}
$$

Similarly, a new set of kernels, denoted as GSO_Z3, can be created by the GSO processing on the weighted and coordinate transformed Zernike polynomials. The GSO_Z3 version of the $14^{\text {th }}$ kernel is shown in Fig 4 (d) when assigning $t=-3$ to the weight function in equation (9). As seen from Fig 4, the GSO_Z3 kernel is now very similar to the strain patterns. Therefore, it is practical to employ the GSO_Z3 kernels to extract shape features from the strain patterns because they are more capable of extracting the essential shape information close to the central hole. Thus, the Zernike moment descriptors of the strain patterns for the loading step 3 as shown in Fig 3 are determined by substituting the GSO_Z3 kernels to equation (7) and its contribution are plotted in Fig 5. Also, the individual kernels of the $2^{\text {nd }}$ to $5^{\text {th }}$ largest terms sorted decreasingly for the strain pattern at $3^{\text {rd }}$ step are shown in Fig 6. It can be seen from Fig 5 that only a very small number of terms are significant. The largest ZMD term is $z_{1}$ which represents the mean value of the shape pattern. The second most significant ZMD is $z_{6}$ which corresponds to the $6^{\text {th }}$ GSO_Z3 kernel as illustrated in Fig 6, representing very closely the maximum and minimum strain patterns around the central hole. Further shape features corresponding to the $27^{\text {th }}, 13^{\text {th }}$ and $14^{\text {th }}$ kernels etc also contain significant shape information.

In order to examine the development of these significant ZMDs in the loading history, more stages within the 3 steps are considered. A total of 13 stages is shown on the horizontal axis of Fig 7. The growth of the 7 largest ZMDs is shown in Fig 7. The ZMD $z_{1}$, representing the average strain, grows rapidly after 2 seconds because the whole specimen begins to yield. The next most dominant shape feature is ZMD $z_{6}$, indicating the distribution of the maximum and minimum strains around the hole, as shown in Fig 7. Several other important shape features $\left\{z_{27}, z_{13}, z_{14}, z_{15}, z_{5}\right\}$ also increase considerably as the stain field develops with increasing load.

Since only a small number of ZMD terms are significant, the number of shape features needed to recover the original pattern may be determined by comparing the norm of the original shape and reconstructed pattern by the retained ZMD terms [7]. The reconstruction from the 10 largest terms is shown in Fig 3. It is seen that the recovered images are very similar to the original patterns. The ratios between the norms of the original and reconstructed strain patterns by 10 terms is $97.2 \%$ meaning that the retained 10 terms are sufficient to recover more than $97 \%$ of the strain pattern.

Therefore, the retrained shape feature terms are succinct and may be assembled as a shape feature vector (SFV) to represent the original shape. Furthermore, the comparison of the full-field 
responses between analytical predictions and experimental measurements can be transferred to the distance measurement between the SFVs [4]. For example, cosine distance may be applied to measure the angular distance of two SFVs.

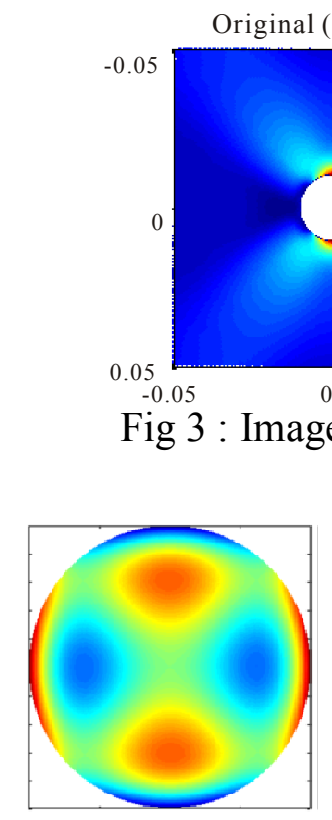

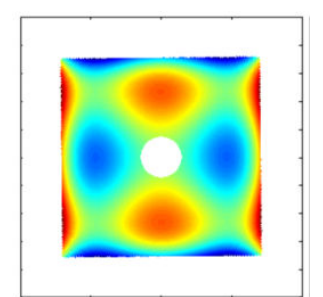

(b) GSO_Z1


of the maximum principal strains for the three loading step3

(a) Circular Z

Fig 4 :

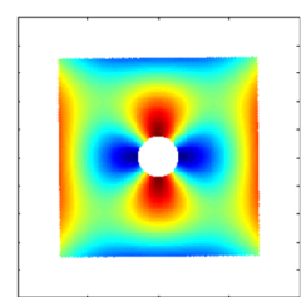

(c) GSO_Z2

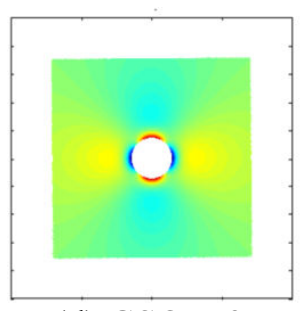

(d) GSO_Z3

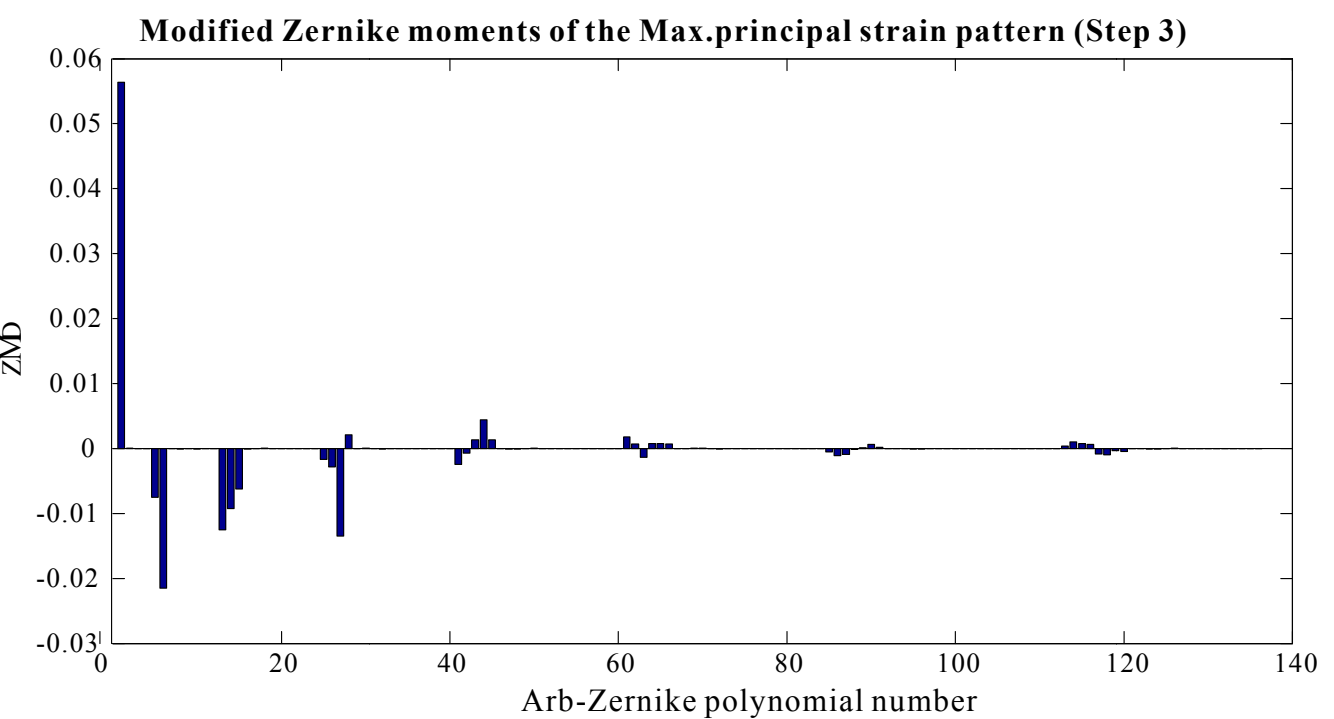

Fig 5 : Shape-descriptor amplitudes - modified Zernike decomposition kernels.
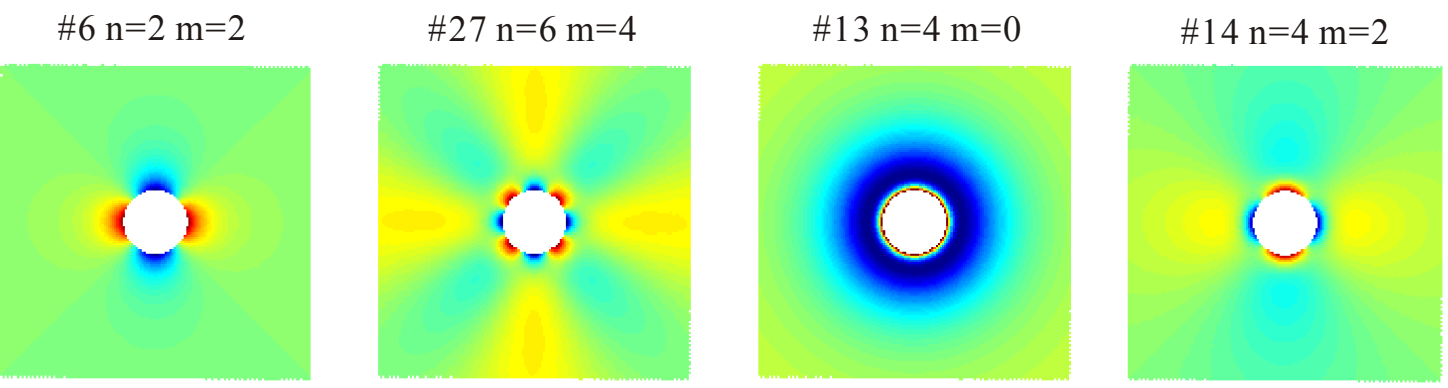

Fig 6 : Individual decomposition kernels (modified Zernike bases) of the strain pattern of step 3 sorted in the decreasing order by the magnitude of the Zernike moment descriptor $\left(2^{\text {nd }}\right.$ to $\left.5^{\text {th }}\right)$. 


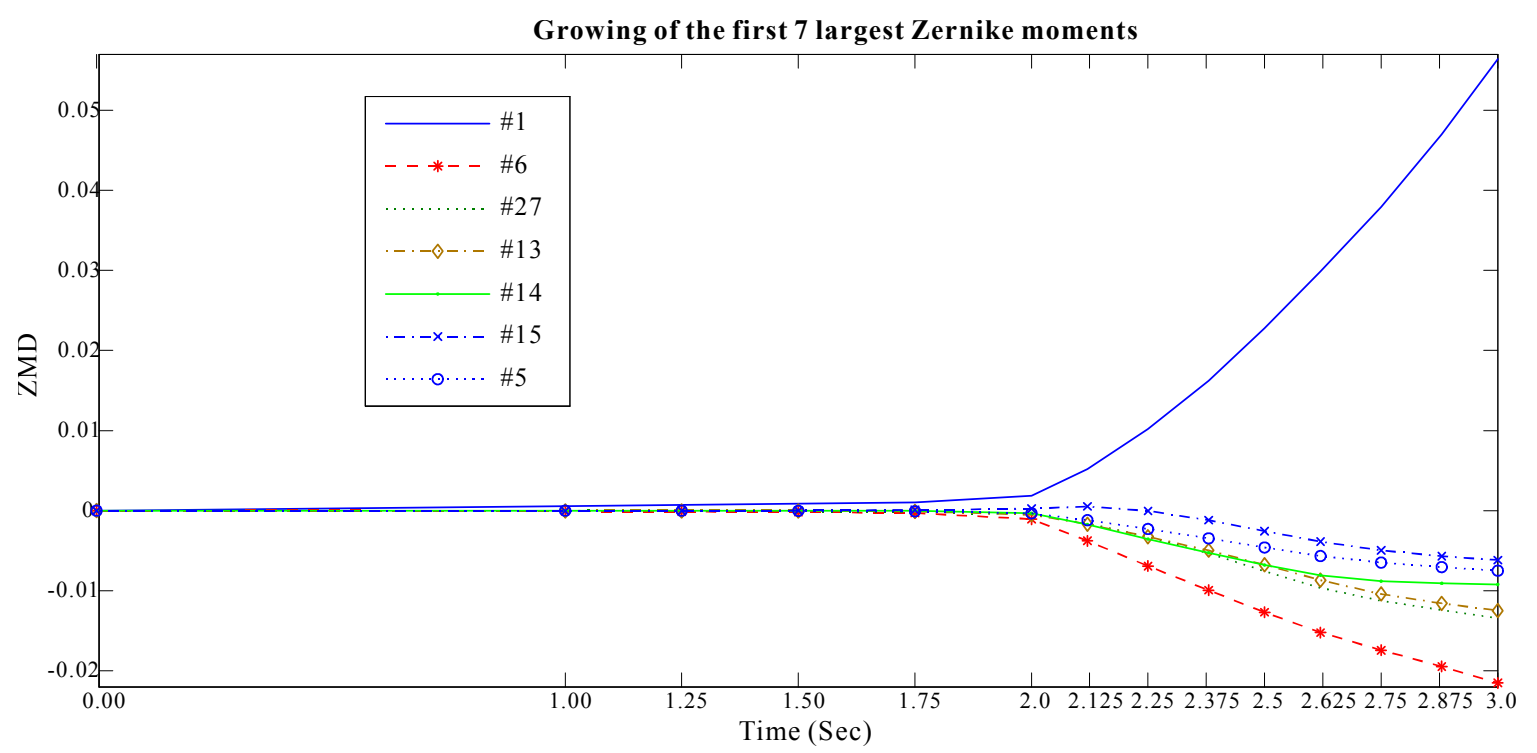

Fig 7 : Development of the 7 largest Zernike moments with loading history -3 seconds

\section{Summary}

Image processing and pattern recognition techniques provide effective and efficient ways to describe the full-field structural responses such as vibration mode shapes and static stress/strain patterns. Shape decomposition based on transformation is one of the most common procedures. Appropriate selection of shape decomposition kernels usually result in a very small number of significant shape features. It is also feasible to construct satisfactory kernels to suit the special problem in hand. Sensitivities of the shape features with respect to the structural parameters were determined. An example problem was presented for demonstration. A modified Zernike moment descriptor was constructed to describe the full-field strain patterns of a square plate with a central circular hole and succinct shape features are obtained. The development of plasticity of the specimen could be reflected by the retained significant shape features.

\section{Acknowledgements}

The authors wish to acknowledge the support of EC project ADVISE (Advanced Dynamic Validations using Integrated Simulation and Experimentation), Grant number 218595.

\section{References}

[1] Mottershead J., and Friswell M., 1993, "Model updating in structural dynamics: a survey," Journal of sound and vibration, 167(2), p. 347-375.

[2] Whelan M. P., Albrecht D., Hack E., and Patterson E. A., 2008, "Calibration of a speckle interferometry full-field strain measurement system," Strain, 44, pp. 180-190.

[3] Wang W., Mottershead J. E., and Mares C., 2009, "Mode-shape recognition and finite element model updating using the Zernike moment descriptor," Mechanical Systems and Signal Processing, 23, pp. 2088-2112.

[4] Wang W., Mottershead J. E., and Mares C., 2009, "Vibration mode shape recognition using image processing," Journal of Sound and Vibration, 326, pp. 909-938.

[5] Mahajan V. N. , 1981, "Zernike annular polynomials for imaging systems with annular pupils," J. Opt. Soc. Am., 71, pp. 75-85.

[6] Wang W., Mottershead J. E., Patterson E. A., and Patki A., "Shape features and finite element model updating from full field strain data," International Journal of Solids and Structures, submitted.

[7] Zernike F., "Translated: diffraction theory of the cut procedure and its improved form, the phase contrast method," Physica, 1. 